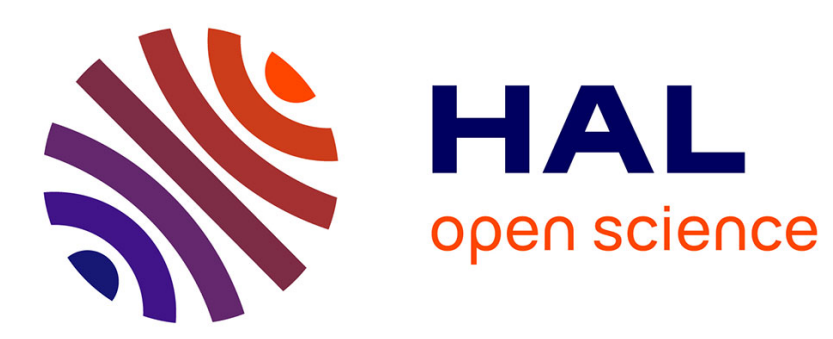

\title{
The Research of Attribute Granular Computing Model in Cognitive and Decision-Making
}

Ruqi Zhou, Yuepeng Zhou

\section{To cite this version:}

Ruqi Zhou, Yuepeng Zhou. The Research of Attribute Granular Computing Model in Cognitive and Decision-Making. 2nd International Conference on Intelligence Science (ICIS), Oct 2017, Shanghai, China. pp.93-103, 10.1007/978-3-319-68121-4_10 . hal-01820916

\section{HAL Id: hal-01820916 https://hal.inria.fr/hal-01820916}

Submitted on 22 Jun 2018

HAL is a multi-disciplinary open access archive for the deposit and dissemination of scientific research documents, whether they are published or not. The documents may come from teaching and research institutions in France or abroad, or from public or private research centers.
L'archive ouverte pluridisciplinaire HAL, est destinée au dépôt et à la diffusion de documents scientifiques de niveau recherche, publiés ou non, émanant des établissements d'enseignement et de recherche français ou étrangers, des laboratoires publics ou privés. 


\title{
The Research of Attribute Granular Computing Model in Cognitive and Decision-making
}

\author{
Ruqi Zhou ${ }^{1,2}$, Yuepeng Zhou ${ }^{2}$ \\ ${ }^{1}$ Department of Computer Science, Guangdong University of Education ,510303, Guangzhou, \\ China \\ ${ }^{2}$ School Of Data and Computer Science,Sun Yat-Sen University,510006, Guangzhou, China
}

\begin{abstract}
The cognitive activities of human beings are complicate and diversified. So far, there hasn't been a universal cognitive model. Each cognitive model generally only represents cognitive features in one or some aspects.Therefore, this paper aims to, based on the granular computing theory and principles, and with attributes and change laws as the main objects of the cognitive course, proposes a cognitive model which not only can describe the thing through attributes to represent the change law, but also can simulate the cognitive decision-making course with respect to the attribute change of the thing. Attribute granular computing , based on qualitative mapping,can simulate the cognitive functions of human brain, such as granulation, organization and causation. Petri net has asynchronous, concurrent and uncertainty characteristics, which is similar to the characteristics of some cognitive activities in human thinking process. Petri net is extended based on the basic concept and logic calculation rules of attribute granular computing in this paper.Some basic elements of a cognitive system, such as knowledge representation, reasoning, learning and memory mode are initially showed in the extended Petri net. The results show that this method can reflect the cognitive process of uncertainty identification and decision-making in a certain extent.
\end{abstract}

Keywords: Cognitive and Decision-making, Attribute Granular Computing, Qualitative Mapping, Fuzzy Set, Petri Net.

\section{Introduction}

Neurophysiological studies indicate that human brain has a dual structure consisting of senses and consciousness, which are two independent brain functions respectively

\footnotetext{
${ }^{1}$ Ruqi Zhou was born in 1971.He is now a Ph.D. candidate in Computer Science and Technology of Sun Yat-sen University.He is also an assistant professor in Guangdong University of Education.His current research interests include machine learning, Intelligent information Processing,Petri net theory \& application,Granular Computing.E-mail address: ruqzhou@163.com(R. Zhou). Yuepeng Zhou was born in 1982.He is now a Ph.D. candidate in Computer Science and Technology of Sun Yat-sen University.His current research areas include Intelligent algorithm, data mining.
} 
processed in two different parts in human brain[1]. According to the structure and physiological functions of the human brain, paper[2] propose a physiology-based cognitive logic theory which holds that the human brain is of a dual structure for senses and consciousness; the sensory memory gets information from the external world, and the consciousness memory gets information from the sensory system; the perception is the conjugation of the sensory information and the consciousness information; and the same transformation exists between the sensory information and the consciousness information. Paper[3] point out that the sense organs only respond to sensory attributes which are sensitive to the sense organs. The information collected by the human brain is the collection of sensory attributes, and the state, movement and change course of a thing is reflected through attributes and the change course of such attributes. The processing of the sensory attributes and their change laws is one of the core problems of the information processing in human brain. The cognitive course of human beings starts from these attributes received through sensory, and an attribute is essentially an information granule.

Paper[4] summarized the human's cognitive ability into: granulation, organization and causation. The concept of the information granule given by paper[5] can be interpreted with an attribute and its qualitative mapping operator, so that the granular computing can be used to simulate a part of the cognitive functions of the human brain.

Decision making is actually a cognitive process.In the decision-making process, people always make decision evaluation systematically from different perspectives in order to obtain more reliable decision results.Qian[7] proposed the multigranulation rough set theory, and proposed multigranulation decision rules which is consistent with human cognition.Song [6] determined the weights of the attributes for the decision objectives through mutual information and conditional entropy, and obtain the efficient sorting results.Yao [8] put forward three-way decision model, which is a common method of human cognitive decision-making. Based on the semantic model of Bayesian decision, a practical and effective decision-making rough set model was given $[9,10]$.Threeway decision has been developed and applied in other areas[11,12].

Feng $[5,13,14]$ put forward a comprehensive decision and evaluation model of attribute coordinate.It is a multi-index factors and multi-attribute decision-making comprehensive evaluation model based on the qualitative mapping. The model is good at simulating the process of psychological cognition of decision maker and gives decision. Its characteristic is that its evaluation method is very close to the brain cognitive thinking mode, which can reflect the cognitive psychological preferences and their change curves of the decision maker.Based on this, this paper describes the brain cognition and decision-making process using the model of attribute granulation.

However, due to the lack of a formal mechanism for the reasoning process of attribute granule, Petri net is used to establish a cognitive decision-making model in this paper.The Petri net is a formalization method with good structure, and is characterized by concurrency, asynchrony, uncertainty and the like. It is similar to certain cognition activity in the thinking course of the human brain. If the places and transitions in the Petri net are simulated with attribute granule and qualitative mapping, the Petri net will 
initially show the characteristics of some basic elements necessary to a cognition system in terms of knowledge representation, knowledge reasoning, decision-making,learning mode, memory mode, etc.This is a new trying.

\section{Qualitative Mapping method in Cognition}

Psychologists believe that human's senses reflect simple attributes of a thing while human's consciousness integrates such simple attributes into comprehensive attributes of the thing. Let $a(x)$ be sensory attributes of a thing $x$. The value of $a(x)$ generally can be divided into quantitative attribute value $d_{\mathrm{a}(\mathrm{x})}$ and qualitative attribute value $P_{a(x)}$. When $x$ has qualitative attribute value $P_{a(x)}$, it is said that the $x$ has the "properties" referred in $P_{a(x)}$. If $P_{a(x)}$ is the property by which the thing $x$ can be distinguished from other things $y$, the $P_{a(x)}$ is called one property or characteristic of thing $x$ [15].

Let $d(x)$ be a quantitative value of the property $p(x)$, and $\left[\alpha_{i}, \beta_{i}\right]$ be a certain qualitative criterion domain of $p(x)$. If $d(x) \in\left[\alpha_{i}, \beta_{i}\right],\left[\alpha_{i}, \beta_{i}\right]$ is a neighborhood of $d(x)[16]$.

If $\left[\alpha_{i}, \beta_{i}\right]$ and $\left[\alpha_{j}, \beta_{j}\right]$ are the qualitative criterions respectively for property $p_{i}(x)$ and property $p_{j}(x)$,we can perform the following logical operations on $\left[\alpha_{i}, \beta_{i}\right]$ and $\left[\alpha_{j}, \beta_{j}\right]$.

(1)Logic not: $\sim\left[\alpha_{i}, \beta_{i}\right], \sim\left[\alpha_{j}, \beta_{j}\right]$.

(2)Logic and: $\left[\alpha_{i}, \beta_{i}\right] \wedge\left[\alpha_{j}, \beta_{j}\right]$.The intersection $\left[\alpha_{i}, \beta_{i}\right] \cap\left[\alpha_{j}, \beta_{j}\right]$, which can be considered as the qualitative criterions for the conjunction property $q(x)=p_{i}(x) \wedge p_{j}(x)$.

Property $p_{i}(x)$ and property $p_{j}(x)$ are homogeneous if a criterion $\left[\alpha_{i j}, \beta_{i j}\right] \in\left[\alpha_{i}, \beta_{i}\right] \cap\left[\alpha_{j}, \beta_{j}\right]$ exists and makes $a(x) \in\left[\alpha_{i j}, \beta_{i j}\right] \wedge b(x) \in\left[\alpha_{i j}, \beta_{i j}\right]$ for any quantitative value $a(x)$ of $p_{i}(x)$ and any quantitative value $b(x)$ of $p_{j}(x)$. Or otherwise, $p_{i}(x)$ and $p_{j}(x)$ are heterogeneous.

(3)Logic or: $\left[\alpha_{i}, \beta_{i}\right] \vee\left[\alpha_{j}, \beta_{j}\right]$.The intersection $\left[\alpha_{i}, \beta_{i}\right] \cup\left[\alpha_{j}, \beta_{j}\right]$ can be considered as the qualitative criterions for the disjunction property $r(x)=p_{i}(x) \vee p_{j}(x)$.

(4)Logical implication: $\left[\alpha_{i}, \beta_{i}\right] \rightarrow\left[\alpha_{j}, \beta_{j}\right]$.

Let $I=\left\{\left[\alpha_{i}, \beta_{i}\right] \mid i \in I\right\}$ be the cluster of all qualitative criterions of a proposition $p$. The mapping $\Psi: \Gamma \rightarrow \Gamma$ is called as the criterion mapping of proposition $p[10]$. If $\left[\alpha_{j}, \beta_{j}\right] \in \Gamma$ exists for any $\left[\alpha_{i}, \beta_{i}\right] \in \Gamma$, then

$$
\Psi\left(\left[\alpha_{i}, \beta_{i}\right]\right)=\left[\alpha_{j}, \beta_{j}\right]
$$

$\Psi$ is called as the qualitaitve criterion transformation from $\left[\alpha_{i}, \beta_{i}\right]$ to $\left[\alpha_{j}, \beta_{j}\right]$,and noted formally as $\left[\alpha_{i}, \beta_{i}\right] \rightarrow\left[\alpha_{j}, \beta_{j}\right]$.

Obviously, homogeneous relations are equivalent based on the criterion mapping, while heterogeneous relations under the criterion mapping can be transformed when certain conditions are satisfied.

Philosophically, the law of quantitative change to qualitative change is represented as the transformation of quantitative properties to qualitative properties. Literatures ${ }^{[12]}$ provide a mathematic model of this cognitive law:

Definition 1[3] Let $a(o)=\stackrel{n}{\wedge} a_{i=1}(o)$ be the integrated attributes of $n$ factor attributes $a_{i}(o), i=1, \ldots, n$, and $x=\left(x_{1}, \ldots, x_{n}\right)$ be the quantitative value of $a(o)$, where: $x_{i} \in X_{i} \subseteq R$ is the quantitative property value of $a_{i}(o), p_{i}(o) \in P_{o}$ is a certain attriibute of $a_{i}(o), \Gamma=\left\{\left[\alpha_{i}, \beta_{i}\right] \mid\right.$ 
$\left[\alpha_{i}, \beta_{i}\right]$ is the qualitative criterion of $\left.p_{i}(o)\right\}$, and hypercube $[\alpha, \beta]=\left[\alpha_{1}, \beta_{1}\right] \times \ldots \times\left[\alpha_{n}, \beta_{n}\right]$ is the qualitative criterion of integrated attribute $p(o)={ }_{i=1}^{n} p_{i}(o)$. The mapping $\tau: X \times \Gamma \rightarrow\{0$, $1\} \times P_{o}$ is called the qualitative mapping $(\mathrm{QM})$ of $x=\left(x_{1}, \ldots, x_{n}\right)$ with $\mathrm{n}$-dimentional hypercube

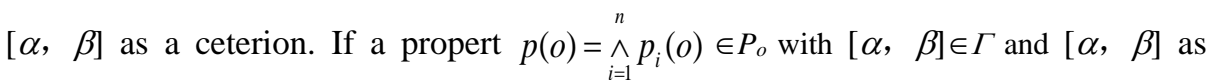
ceterion exists for any $x \in X$, then:

$$
\tau(x,[\alpha, \beta])=\widehat{\wedge}_{i=1}^{n}\left(x_{i} \in\left[\alpha_{i}, \beta_{i}\right]\right)=\hat{}_{i=1}^{n} \tau_{p_{i}(o)}\left(x_{i}\right)
$$

Where,

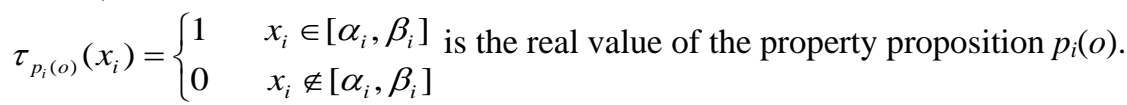

The numerical domain $d_{a(x)}$ of attribute $a(x)$ of the thing $\mathrm{x}$ and the criterion domain cluster $\Gamma=\left\{\left[\alpha_{i}, \beta_{i}\right] \mid i \in I\right\}$ form a criterion topological space $T\left(d_{a(x)}, \Gamma\right)$. Therefore, the definition 1 actually is a cognitive mode of the granular computing done in the criterion topological space.

\section{Attribute Granules in Cognitive and decision-making}

A granule is not only a cluster or a collection of entities, but also the abstraction of such cluster or collection[17],[18]. If all attributes of a thing is considered as a whole, a certain attribute can also be considered as a granule. The qualitative mapping, which creates maps from an attribute to a property, is an intuitive attribute computing but also an intuitive granular computing. Attributes are closely related to the qualitative criterion, or in other words, attributes are closely related to the qualitative mapping. When we say a thing $\mathrm{x}$ has the property $p(x)$ referred in $P_{\mathrm{a}(\mathrm{x})}$ (the quantity property of an attribute $a(x)$ ), what we really mean is the thing has the property $p(x)$ under certain qualitative criterion. Therefore, an attribute with a bigger structure intuitively composed of attributes which contain qualitative criterion or qualitative mapping is called an attribute granule.

An attribute granule in the attribute topological space can be expressed as a 2-tuple:

$$
(P, \Gamma)=\left(p_{i}(x),\left[\alpha_{i}, \beta_{i}\right]\right), \text { or }(P, \Gamma)=\left(d_{a(x)},\left[\alpha_{i}, \beta_{i}\right]\right)
$$

Where, $a(x)$ is a sensory attribute of a thing $\mathrm{x}, p_{\mathrm{i}}(x)$ is the property of $a(x),\left[\alpha_{\mathrm{i}}, \beta_{\mathrm{i}}\right]$ is a qualitative criterion domain, $d_{\mathrm{a}(\mathrm{x})}$ is a quantitative attribute value of $a(x)$, and a granule $\left(p_{\mathrm{i}}(x),\left[\alpha_{\mathrm{i}}, \beta_{\mathrm{i}}\right]\right)$ is referred to as $\Gamma_{\mathrm{i}}\left(p_{\mathrm{i}}\right)$ for short. Formally, an attribute granule sometimes can also be equivalent to a first-order predicate formula.

Definition 2 Let $\Gamma\left(p_{\mathrm{i}}\right)$ and $\Gamma\left(p_{\mathrm{j}}\right)$ be two attribute granules. The computing formula of $\Gamma\left(p_{\mathrm{i}}\right)$ and $\Gamma\left(p_{\mathrm{j}}\right)$ with respect to logic connector can be defined as follows:

(1) $\sim \Gamma\left(p_{\mathrm{i}}\right)=\left(\sim p_{\mathrm{i}}, \Gamma^{\prime}\right)$, where, $\Gamma^{\prime}$ is the complementary operation of the qualitative criterion of $\Gamma$;

(2) $\Gamma_{\mathrm{i}}\left(p_{i}\right) \wedge \Gamma_{j}\left(p_{j}\right)=\left(p_{i}(\mathrm{x}) \Delta p_{j}(\mathrm{x}),\left[\alpha_{\mathrm{i}}, \beta_{\mathrm{i}}\right] \cap\left[\alpha_{\mathrm{j}}, \beta_{\mathrm{j}}\right], \Psi\left(\Gamma_{\mathrm{i}}\right)=\Gamma_{j}\right)$.

(3) $\Gamma_{\mathrm{i}}\left(p_{i}\right) \vee \Gamma_{j}\left(p_{j}\right)=\left(p_{i}(\mathrm{x}) \nabla p_{j}(\mathrm{x}),\left[\alpha_{\mathrm{i}}, \beta_{\mathrm{i}}\right] \cup\left[\alpha_{\mathrm{j}}, \beta_{\mathrm{j}}\right], \Psi\left(\Gamma_{\mathrm{i}}\right)=\Gamma_{j}\right)$. 
(4) $\Gamma_{\mathrm{i}}\left(p_{i}\right) \rightarrow \Gamma_{j}\left(p_{j}\right)=\left(p_{i}(\mathrm{x}) \Lambda p_{j}(\mathrm{x}),\left[\alpha_{\mathrm{i}}, \beta_{\mathrm{i}}\right] \rightarrow\left[\alpha_{\mathrm{j}}, \beta_{\mathrm{j}}\right], \Psi\left(\Gamma_{\mathrm{i}}\right)=\Gamma_{j}\right)$.

Where, $\nabla$ is attribute disjunction, $\Delta$ is attribute conjunction, $\Lambda$ is attribute integration or reasoning, and $\Psi$ is qualitative criterion transformation.

Definition 3 A formula $\left(\Gamma_{\mathrm{i}}\left(p_{\mathrm{i}}\right)\right)$ is true if and only if the true value of its transformation function $\eta\left(\Gamma_{\mathrm{i}}\left(p_{\mathrm{i}}\right)\right)$ is 1 or above 0.5 .

Definition 4 The logical formula of an attribute granule can be interpreted semantically as:

A quadruple (4-tuple) D:

$D=\left\{U, \Psi, \eta,\left\{\Gamma_{\mathrm{i}}, \quad i \in I\right\}\right\}$

(1) $U$ is a non-empty set called domain of interpretation (DOI);

(2) $\Psi$ is a mapping and $P: U \rightarrow U$;

(3) For any $i \in I, \eta: \mathrm{U} \times \Gamma \rightarrow[0,1]$ is a degree function;

(4) $\Gamma_{\mathrm{i}}$ is the qualitative criterion domain.

In the interpretation $D$, each qualitative criterion transformation formula $P$ corresponds to an element $v(P)$ in $[0,1]$, which is called the true value of qualitative criterion transformation formula $P$ in interpretation $\mathrm{D}$, where:

(1) If $P$ is the logical formula of the attribute granule $\left(\Gamma_{\mathrm{i}}\left(p_{\mathrm{i}}\right)=\left(p_{\mathrm{i}}(x),\left[\alpha_{\mathrm{i}}, \beta_{\mathrm{i}}\right]\right)\right.$, then:

${ }_{V}\left(p_{i}(x),\left[\alpha_{i}, \beta_{i}\right]\right)=\left\{\begin{array}{lll}1 & \text { iff } & x \in\left[\alpha_{i}, \beta_{i}\right] \\ \neg & \text { iff } & x \notin\left[\alpha_{i}, \beta_{i}\right]\end{array}\right.$

(2) If $P$ is the logical formula of the attribute granule $\sim G$, then $v(\sim G)=(v(G))^{\prime}=v\left(\sim p_{\mathrm{i}}(\mathrm{x}), \Gamma-\left[\alpha_{\mathrm{i}}, \beta_{\mathrm{i}}\right]\right) ;$

(3) If $P$ is the logical formula of the attribute granule $G \vee H$, then $v(G \vee H)=v\left(p_{\mathrm{i}}(\mathrm{x}) \nabla p_{\mathrm{j}}(\mathrm{x}),\left[\alpha_{\mathrm{i}}, \beta_{\mathrm{i}}\right] \cup\left[\alpha_{\mathrm{j}}, \beta_{\mathrm{j}}\right]\right) ;$

(4) If $P$ is the logical formula of the attribute granule $G \wedge H$, then $v(G \wedge H)=v\left(p_{\mathrm{i}}(\mathrm{x}) \Delta p_{\mathrm{j}}(\mathrm{x}),\left[\alpha_{\mathrm{i}}, \beta_{\mathrm{i}}\right] \cap\left[\alpha_{\mathrm{j}}, \beta_{\mathrm{j}}\right]\right) ;$

(5) If $P$ is the logical formula of the attribute granule $G \rightarrow H$, then $v(G \rightarrow H)=v\left(p_{\mathrm{i}}(\mathrm{x}) \Rightarrow p_{\mathrm{j}}(\mathrm{x}),\left[\alpha_{\mathrm{j}}, \beta_{\mathrm{j}}\right]=\Psi\left(\left[\alpha_{\mathrm{i}}, \beta_{\mathrm{i}}\right]\right)\right)$

Where, $\Rightarrow$ is attribute reasoning.

Definition $5 G$ and $H$ in the logical formula of the attribute granule are called equivalence. If the assignment of any interpretation $\mathrm{D}$ is $v(G)=v(H)$, then $G=H$.

Definition $6 \Gamma_{\mathrm{i}}\left(p_{\mathrm{i}}\right) \rightarrow \Gamma_{\mathrm{i}}\left(p_{\mathrm{i}}\right)$ is an interference in the logic of the attribute granule, which means that there is a mapping $\Psi_{\mathrm{ij}}: \Gamma \rightarrow \Gamma$ to make $\Psi_{\mathrm{ij}}\left(\left[\alpha_{\mathrm{i}}, \beta_{\mathrm{i}}\right]\right)=\left[\alpha_{\mathrm{j}}, \beta_{\mathrm{j}}\right]$ if $\left(p_{\mathrm{i}}(x) \Rightarrow p_{\mathrm{j}}(x)\right)$.

\section{Cognitive decision-making and fuzzy attribute granule}

Definition 7[5] A mapping $\eta: X \times \Gamma \rightarrow[-1,1]_{i}$ is a function reprenting the transformation degree of quality property set $p_{i}\left(\xi_{i}\right)$. If $\exists \eta_{i}(x) \in[-1,1]_{i}$ for $\forall\left(x, N\left(\xi_{i}, \delta_{i}\right)\right) \in X \times \Gamma$, then: $\eta\left(x, \xi_{I}, \delta_{i}\right)=\left|x-\xi_{i}\right| \perp \delta_{i}=\eta_{i}(x)$

Where, The $N\left(\xi_{i}, \delta_{i}\right)$ is the qualitative criterion domain, $\delta_{I}$ is radius of the domain, $\xi_{I}$ is core of the domain, $\eta_{i}(x)$ is diversity factor of $\left|x-\xi_{i}\right|$ and $\delta_{i}$ as to the mathematics nature. 
Zadeh studied the size of divided class or granule and defined the information granularity as a proposition: value of $x$ belongs to the fuzzy subset $G \subseteq U$ by membership $\lambda$. The $\mathrm{x}$ is a variable of $U$ and the value of $x$ is an entity of $U$, represented by $g=x$ is $G$ is $\lambda$, and noted formally as:

$g=\{u \in U$ : value of $x(v(x)=u, v$ is assignment symbol on $U)$ is calculated based on membership of $\lambda$ in the fuzzy set $G \subseteq U$. .

Obviously, in the formulation above, $0 \leq \lambda \leq 1$. Based on fuzzy set theory, $\lambda$ is a fuzzy membership function. Based on logic, the $\lambda$ is the fuzzy truth value or probability of the proposition.

It is easy to figure out by comparison that: under the qualitative mapping $\tau$ with transformation program function, a information granule $\left(p_{i}(x),\left[\alpha_{i}, \beta_{i}\right]\right.$ or $\left(d_{a(x)},\left[\alpha_{i}, \beta_{i}\right]\right)$ in Definition 1 shows that 'the fact attribute $a(x)$ having the property $p_{\mathrm{i}}(x)$ is calculated based on the membership of the attribute value $d_{a(x)}$ in the criterion $\left[\alpha_{i}, \beta_{i}\right]^{6}$. They are the same in essence.

Definition 8 Let the criterion domain $N_{i}\left(o_{i}, r_{i}\right)$ be the core of a fuzzy set A, namely $N_{i}\left(o_{i}, r_{i}\right)=A_{1}=\left\{x \mid \mu_{A}(x)=1\right\}$. The $\mu_{A}(x)$ is membership of $x \in A$. $\lambda$ cut set of $\mathrm{A}$ is $A_{\lambda}=\{x$ $\left.\mid \mu_{A}(x) \geq \lambda\right\}, \quad \lambda \in(0,1], \lambda \in(0,1]$, consisting of element $\mathrm{x}$ of membership not less than $\lambda$. $\Psi \lambda$ is the $\lambda$ criterion transformation of the fuzzy set A whose core is $N_{i}\left(o_{i}, r_{i}\right)$ if it meets following equation:

$$
\begin{gathered}
\Psi_{\lambda}\left(N_{i}\right)=A_{\lambda} \\
\Psi_{\alpha}\left(\left[\alpha_{i}, \beta_{i}\right]\right)=N\left(T_{\alpha}\left(o_{i}\right), \Psi_{\alpha}\left(r_{i}\right)\right)=N_{\alpha}\left(o_{k}, r_{k}\right)
\end{gathered}
$$

Definition 9 Let A and B be two fuzzy sets with criterion domains $\left[\alpha_{i}, \beta_{i}\right]$ and $\left[\alpha_{k}\right.$, $\left.\beta_{k}\right]$ as cores respectively. $\Psi_{\alpha}$ is the criterion transformation from $\left[\alpha_{i}, \beta_{i}\right]$ to $\left[\alpha_{k}, \beta_{k}\right]$ if it meets following equation:

$$
\Psi_{\alpha}\left(\left[\alpha_{i}, \beta_{i}\right]\right)=N\left(\Psi_{\alpha}\left(o_{i}\right), \Psi_{\alpha}\left(r_{i}\right)\right)=N_{\alpha}\left(o_{k}, r_{k}\right)
$$

The following theorem can be obtained immediately:

Based on the definition 10,criterion transformation $\Psi_{\alpha \lambda}$ as the composite transformation of $\Psi_{\alpha}$ and $\lambda$-criterion transformation if it meets conditions below:

$$
\Psi_{\alpha \cdot \lambda}\left(N_{\mathrm{i}}\right)=\Psi_{\lambda} \cdot \Psi_{\alpha}\left(N_{\mathrm{i}}\right)=\Psi_{\lambda}\left(N_{\alpha}\right)=N_{\lambda}\left(o_{\alpha}, r_{\alpha}\right)
$$

The mapping $\eta: X \times \Gamma \rightarrow[-1,1]$ is the transformation degree function for $p_{\mathrm{i}}(x)$ to reflect its nature characteristics; if $\forall\left(x, N\left(\xi_{\mathrm{i}}, \delta_{\mathrm{i}}\right)\right) \in X \times \Gamma, \exists \eta_{\mathrm{i}}(x) \in[-1,1]$, which makes

$$
\eta\left(x, \xi_{\mathrm{i}}, \delta_{\mathrm{i}}\right)=\left|x-\xi_{\mathrm{i}}\right| \perp \delta_{\mathrm{i}}=\eta_{\mathrm{i}}(x)
$$

Where, $\eta_{\mathrm{i}}(x)$ is the variability between $\left|x-\xi_{\mathrm{i}}\right|$ and $\delta_{1}$.

\section{Set up cognition formal model with attribute granule}

The transition nodes in Petri net can be viewed as a qualitative mapping, and the quantity properties, quality properties and qualitative criterion domain mapping of the attributes of a thing care places in the Petri net. Therefore, if the place nodes are extended into an information granule, the transition nodes will naturally be the granular transformation mapping. The definition of ordinary Petri net is extended below, and is considered as a cognitive system model. 
Definition 10 The form of a cognitive system can be defined as a nonuple (10-tuple) Petri net:

$\mathrm{CSPT}=\left\{P, T, F ; M_{0}, S, O, N, W, \Psi, \Gamma\right\}$

Where, $P$ is a finite set of place nodes of attribute granules; $T$ is a finite set of transition nodes of attribute granules, equivalent to a granular transformation; and $F$ is a marked relation on $P \times T$, representing the connections of place nodes to transition nodes, the rated input and the input strength calculation function $S$ on connection line, as well as the corresponding connection strength. $M_{0}$ is a function defined on $P$ with its value range being $[0, K]$ ( $\mathrm{K}$ is a bounded real number), indicating the initial marking of the place nodes at the beginning of the operation; $N$ is the output strength function at a qualitative level; $O$ is the operation of m-dimensional weighted qualitative operator; $\Psi$ is the criterion transformation, $\Gamma$ is qualitative criterion; and $W$ is the attribute weight, a function of $\Gamma$.

If the number of input link in any place node is 0 , the place node is called as the input node of RS. If the number of the output link $n$ any place node is 0 , the place node is called as the output node of CSPT.

CSPT is mainly characterized in that:

(1) The starting threshold of transition nodes of CSPT is the detection threshold of sensory nerves, and the starting course is the transformation of the qualitative mapping degree.

(2) Directed edges in CSPT can be divided into input link and output link. For any node, the link pointing to it is called its input link and that deviating from it is called its output link. The link strength is attached to the input and output links.

(3) The capacity of place node is a bounded real number and the start-up of transition node depends on whether value of the input of quantitative (or qualitative) property of attribute on the input link, connection strength and the degree function of qualitative mapping (which is called input strength) is higher than the starting threshold of the transition node.

This is an operable net. The logical relationship between place nodes can be represented as shown in Fig.1 to Fig.3. Decision problems described in the qualitative mapping model can be represented by CSPT in a vivid and visual manner.

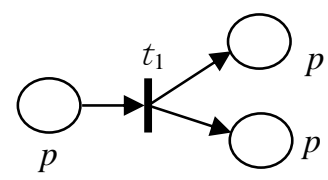

Fig. 1 One Cause to Multiple Effects

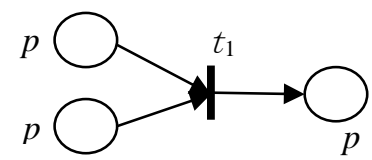

Fig.3 Multiple Causes to One Effect

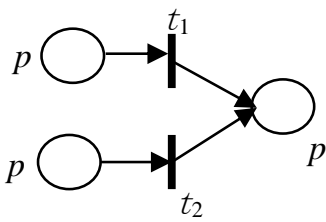

Fig.2 One Cause to Multiple Effects 


\section{Decision-making in CSPT}

Paper[19-20] proposed a logical structure of memory which holds that the sensory storage in the three-stage processing model for memory information is merely an inputoriented storage. In view of the whole process from input to output of the sensation, consciousness and the qualitative mapping, paper[21] indicate that the sensation is a test response to all simple attribute properties of an object, and that the consciousness is responsible for integrate them to form the overall consciousness of human brain to the object. The pattern recognition of the outer attribute information granules through sensation and consciousness can be represented with the following classification function:

$$
s_{y}\left(p_{i}(\mathrm{x}),\left[\alpha_{\mathrm{i}}, \beta_{\mathrm{i}}\right]\right)=s_{y} \Delta \Gamma\left(p_{i}\right)=\left\{\begin{array}{cc}
s_{y}(x, y) & \text { If } s_{y}(x) i s \text { a simple factor of } p_{i}(x) \\
-s_{y}(x, y) & \text { or }
\end{array}\right.
$$

Where, $\left(p_{i}(x),\left[\alpha_{i}, \beta_{i}\right]\right)$ and $\Gamma\left(p_{\mathrm{i}}\right)$ represent the attribute granule of a thing $x, s_{y}$ is sensory neuron which can be considered as the mapping of memory set $M(x, y)$ of the thing $x$ from attribute set $P_{x}$ to a subject $y$. Namely, $s_{y}: P_{x} \rightarrow M(x, y) . \Delta$ is an extraction operation.

The representation of attribute granules in CSPT is more natural, and the whole process of knowledge representation reflects the reasoning course of thinking operation in the human brain.

Assume the following thinking operation exists, $M(y)$ is the memory set of a subject $y$, and $s_{i}(y) \in M(y)$ is detecting neurons (or sensor or the like) of the attribute $s_{i}(x)$ of a thing $x$. Let $P_{x}=\left\{p_{j}(x) \mid j=1, \ldots, n\right\}$ be the set of the attribute granules of $x$, and $p(x)=\wedge p_{j}(x)$ be the integration of $r(r \leq n)$ attributes of $x$, where, $\wedge$ is integration operator. If $r=n$ and $T(x)=\bigwedge p_{j}(x)$ is the integrated (comprehensive) attributes of $x$, then, the attribute detection on $T(x)$ by sensory neuron $s_{i}(y)$ can be represented as a mapping $s_{i}(y): P_{x} \rightarrow M(y)$ from $P_{x}$ to $M(y)$, and:

$$
s_{i}(y)(T(x))=s_{i}(y) \Delta\left(p_{i}(x),\left[\alpha_{i}, \beta_{i}\right]\right)=s_{i}(x, y)
$$

$s_{i}(x, y) \in M(y)$ is the sensory image of $s_{i}(x)$ in $M(y)$. the above formula indicates that: if $T(x)$ contains attributes $s_{i}(x)$ which can be detected by $s_{i}(y)$, then $s_{i}(y)$ will decompose and extract $s_{i}(x)$ from $T(x)$, and store the detection image $s_{i}(x, y)$ in $M(y)$. or otherwise, $s_{i}(y)$ will tell the computer that $x$ doesn't have attribute $s_{i}(x)$. The decisionmaking process of the above thinking operation can be represented in CSPT as shown in Fig. 4.

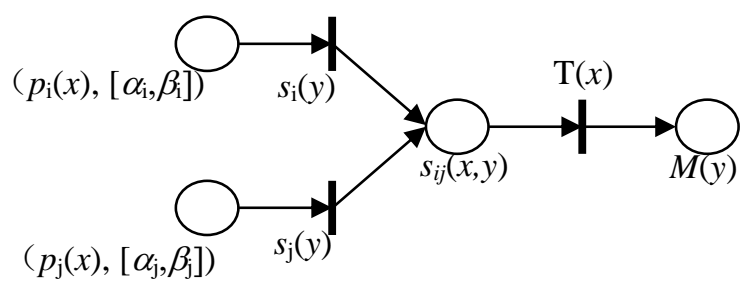

Fig.4 Decision-making in CSPT 


\section{Conclusion}

Sense organs of human being receive all kinds of sensory attribute information and respond accordingly. A thing represents its state, movement and change with its attributes and the attribute change courses. The cognitive process of human beings are closely related such attribute information received. Philosophical opinions hold that attribute not only expresses the quality property which needs to be expressed, but also has quantity property which needs to be limited and regulated. Based on such philosophical opinions in combination with the theories with respect to granular computing and qualitative criterion transformation, , this paper provides the basic mathematical model of the cognition and the definition of Petri net. However, the cognitive process of human beings is very complicated. This paper gives merely the framework of a basic cognitive mode. More study work needs to be done.

\section{Acknowledgments}

This work is supported by the National High Technology Research and Development Program of China (863) sub project(2012AA101701-06), the Guangdong Science and Technology Plan Project(2012B010100049), the Characteristic Innovation Project of Guangdong Universities(natural science)(2014KTSCX195), open fund of Fujian Provincial Key Laboratory of Big Data Mining and Applications(Fujian University of Techology,2017).

\section{References}

1. Rajendra D. Badgaiyan.Neuroanatomical Organization of Perceptual Memory:An fMRI Study of Picture Priming[J]. Human Brain Mapping,10:197-203,(2000)

2.Peking University Bio Intelligent Technology Research Group.The fifth generation computer and its cognitive logic methodology,Frontire science,1(1):18-23,(2007)

3.J Feng,Z Dong,A Mathematical model of perception schema generation and recognition based on attributive in tegration,Computer research \& development,34(7):487-491,(1997)

4.Zadeh L A.Towards a theory of fuzzy information granulation and its centrality in human reasoning and fuzzy logic.Fuzzy Sets and Systems.19:111-127(1997)

5.Jiali Feng. Attribute network computing based on qualitative mapping and its applications in pattern recognition. Journal of Intelligent \& Fuzzy Systems, 19(2):1-16(2008)

6.P. Song, J. Y. Liang, Y. H. Qian. A two-grade approach to ranking interval data. KnowledgeBased Systems. 27:234-244(2012)

7.Y. H. Qian, J. Y. Liang, Y. Y. Yao, C. Y. Dang. MGRS: a multigranulation rough set .Information Sciences. 180:949-970(2010)

8. Y. Y. Yao. The superiority of three-way decisions in probabilistic rough set models. Information Sciences. 181(6):1080-1096(2011)

9.Y. Y. Yao. Three-way decisions with probabilistic rough sets. Information Sciences. 180(3):341-353(2010)

10.Sun, Bingzhen; Ma, Weimin; Qian, Yuhua,Multigranulation fuzzy rough set over two universes and its application to decision making,Knowledge-Based Systems, 123: 61-74,(2017)

11.D. Liu, Y. Y. Yao, T. R. Li. Three-way investment decisions with decision theoretic rough sets.International Journal of Computational Intelligence Sys tems. 4(1):66-74(2011) 
12.Nauman, Mohammad; Azam, Nouman; Yao, JingTao,A three-way decision making approach to malware analysis using probabilistic rough sets, Information Sciences, 374: 193-209,(2016).

13.J.Feng, Qualitative mapping, linear transformation of qualitative criterion and artificial neuron,Proc. Of the IJCAI-2007,workshop theme:complex valued neural networks and neurocomputing:novel methods,applications and implementations,Hyderabad,India,January 612(2007)

14.J.Feng,Z.Wu, Conversion degree functions induced by qualitative mapping and artificial neurons, Proceedings of ICMLC2003,IEEE 03EX693,1135-1140(2003)

15.J.Feng,Z.Dong.A Mathematical model of sensation neuron detection based on attributive abstraction and integration.Computer research \& development,34(7):481-486(1997)

16.J.Feng.Granular transformation of qualitative criterion, orthogonality of qualitative mapping system and pattern recognition,Proceeding of 2006 IEEE international conference on granular computing .Atlanta,Georgia,USA:10-12(2006)

17.Zhong, Chunfu; Pedrycz, Witold; Wang, Dan;Granular data imputation: A framework of Granular Computing,Applied Soft Computing, 46: 307-316,(2016)

18. Wolski, Marcin; Gomolinska, Anna, Rough Granular Computing in Modal Settings: Generalised Approximation Spaces,Fundamenta Informaticae, 148: 157-172,(2016)

19.Wang Yingxu, Wang Ying. Cognitive Informatics Models of the Brain. IEEE Transactions on Systems, Man and Cybernetics,36(2):203-207(2006)

20.Y Wang,Y Qi,Memory-Based cognitive modeling for visual information processing,PR \& AI,24(2):144-150,(2013)

21.Z Dong,J Feng,An Attributive coordinate representation of schematic memory,Computer research \& Development,35(8):694-698,(1998) 